\title{
Manejo ambulatorio del paciente con diabetes en tiempos de COVID-19
}

\author{
In the SARS-CoV-2 pandemic: Outpatient management of the diabetes \\ patient
}

\author{
Ramírez-Rincón $A^{1}$, Builes-MontañoCE ${ }^{2}$
}

${ }^{1}$ Clínica Integral de Diabetes (CLID). Profesor Titular, Universidad Pontifica Bolivariana. Miembro de número, Asociación Colombiana de Endocrinología, Diabetes y Metabolismo.

${ }^{2}$ Miembro de número, Asociación Colombiana de Endocrinología, Diabetes y Metabolismo. Grupo de Diabetes, Hospital Pablo Tobón Uribe. Profesor Asociado, Sección de Endocrinología y Metabolismo, Universidad de Antioquia.

Autor de correspondencia: Alex Ramírez-Rincón

Correo electrónico: alexramirezrincon@gmail.com

\section{Resumen}

El tratamiento ambulatorio de pacientes con enfermedades crónicas no transmisibles (ECNT) ofrece retos interesantes en el contexto de la pandemia por SARS-CoV-2 (COVID-19), que exigen un análisis individual de la diabetes. Es a lugar discutir elementos del tratamiento ambulatorio: desde implementación de modelos específicos, recomendaciones de cambios en el estilo de vida, orientación en comorbilidades, identificación de factores de riesgo adicionales, hasta sugerencias en cada uno de los grupos farmacológicos que acá competen. Todo lo anterior con el propósito de brindar el mejor soporte a los pacientes en el panorama no hospitalario.

Palabras clave: diabetes mellitus, COVID-19, tratamiento.

\section{Abstract}

Outpatient treatment of patients with Chronic Noncommunicable Diseases (CNCD) offers interesting challenges in the context of the SARS-CoV-2 (COVID-19) pandemic, thus Diabetes requires an individual analysis. It is a place to discuss elements of outpatient treatment: from implementation of specific models, recommendations for changes in lifestyle, orientation in comorbidities, identification of additional risk factors, to suggestions in each of the pharmacological groups that are involved here. All of the above in order to provide the best support to patients in the non-hospital setting.

Keywords: Diabetes mellitus, COVID-19, Treatment.

\section{Introducción}

El manejo ambulatorio de pacientes con enfermedades crónicas no transmisibles (ECNT) ofrece retos interesantes en el contexto de una pandemia, desde la adaptación precoz a la nueva realidad, hasta las directrices preventivas propias de un fenómeno infeccioso de las magnitudes actuales del SARS-CoV-2 (COVID-19). Es el caso, entonces, de la diabetes, que como patología metabólica de gran prevalencia exige una mirada acuciosa con múltiples aspectos en el manejo ambulatorio, que se traerán a colación con mayor o menor peso en la evidencia clínica según cual sea el caso.

Inicialmente, es apropiado destacar el beneficio de consolidar controles médicos ambulatorios a través del uso de modelos virtuales en salud (telemedicina-telesalud). De manera paralela, cuando sea necesaria la consulta presencial y/o las situaciones de confinamiento se liberen progresivamente, se deberán cumplir las recomendaciones de la Organización Mundial de la Salud (OMS), los Centros para el Control y la Prevención de Enfermedades (CDC) o demás entes locales (como el Instituto Nacional de Salud de Colombia) que orienten el distanciamiento y las medidas preventivas en lugares de atención médica ambulatoria ${ }^{(1,2)}$.

Durante la actual pandemia, en el grupo de pacientes con trastornos en el metabolismo de los carbohidratos (TMCHO) y aquellos con diagnóstico de diabetes mellitus tipo 1 (DM1) o DM2 que no han sido infectados por la COVID-19, sería a lugar intensificar el control metabólico y seguimiento con el objetivo de evitar estados de hiperglucemia, este aspecto orientado a la disminución de la predisposición aguda de procesos infec$\operatorname{ciosos}^{(2,3)}$. De la misma manera, las recomendaciones de un buen estilo de vida y todo aquello concerniente a la adherencia al plan de alimentación, la actividad física y el suministro adecuado del tratamiento farmacológico, serán pilares fundamentales del control ambulatorio ${ }^{(4)}$.

Ratificando la importancia del enfoque holístico en estos pacientes, es prudente ser enfáticos en el cumplimiento de las 
metas de los demás factores de riesgo, como colesterol LDL, presión arterial y obesidad ${ }^{(4)}$.

Aun dirigiendo al lector a las guías pertinentes para cada factor, en este sentido y de manera puntual se destaca que el uso de inhibidores de la enzima convertidora de la angiotensina (IECA) no debe ser modificado, dado que no hay evidencia suficiente para sugerir su suspensión y/o cambio por otros antihipertensivos o nefroprotectores ${ }^{(5)}$. En cuanto al uso de estatinas, dada la evidencia que muestra un aumento en la expresión de la enzima convertidora de la angiotensina 2 (ECA2) con su uso, se sugiere que podrían aumentar el riesgo de infección y mortalidad por COVID-19; sin embargo, no existe evidencia fuerte de que esto tenga un impacto clínico, así que no deben suspenderse debido a los ya conocidos beneficios a largo plazo y la posibilidad de aumentar el riesgo de una tormenta de citocinas por el incremento súbito de la interleucina 6 (IL-6) y la IL-1B ${ }^{(6,7)}$.

Dada la prevalencia de obesidad como comorbilidad de alta asociación con la DM2, es un factor no menor que debe tenerse en cuenta; además, ha sido identificada como factor de riesgo y gravedad de enfermedad por coronavirus independiente de diabetes, motivo por el cual el énfasis en las directrices para el control de peso en estos pacientes toma mayor trascendencia.

En cuanto al control glucémico en la fase preventiva de la infección por COVID-19, se corroboran las metas ya conocidas. La evidencia es precaria si buscamos metas específicas para la infección en curso, por lo cual algunas publicaciones sugieren puntos de corte fundamentados en consenso de expertos o de sociedades científicas: glucemia basal, 72$144 \mathrm{mg} / \mathrm{dL}$ (pacientes "frágiles" o ancianos: 90-144 mg/dL); HbA1c, $<7 \%$, con uso de monitorización continua de la glucosa (MCG); TIR, > 70\% (>50\% en pacientes "frágiles" y ancianos); e hipoglucemia, $<4 \%$ ( $<1 \%$ en pacientes "frágiles" y ancianos) $(1,8)$. En este mismo sentido, en el contexto ambulatorio, no se recomienda aumentar la frecuencia de la automonitorización. Lo que sí es susceptible de destacar es la ventaja de los dispositivos de tipo MCG intermitente (MCGi) para establecer unas mejores métricas de glucemia del proceso en curso ${ }^{(1,2)}$; cabe anotar que esta sugerencia de manejo aplica para los pacientes con DM1 y DM2; en ambos casos, la evidencia en el control es sólida ${ }^{(1-3)}$. En pacientes con antecedentes de TMCHO tipo prediabetes, que cursen con infección por COVID-19 (con o sin ameritar manejo intrahospitalario), se sugiere intensificar la frecuencia de seguimiento dada la probable asociación descrita a la fecha entre la disminución en la población de células beta (dados los receptores de ECA-2) y la progresión de esta condición clínica, lo cual ocasionaría un aumento de la hiperglucemia de base ${ }^{(1,3)}$.

\section{Consideraciones especiales en el manejo farmacológico ambulatorio}

Sin perder de vista el objetivo fundamental de mantener el buen control glucémico en el contexto de fase, además de la necesidad de optimización en caso de diagnóstico de infección por COVID-19, es importante denotar consideraciones especiales en cuanto a la continuidad del manejo farmacológico ambulatorio.

\section{Biguanidas}

Si bien son conocidas las postulaciones de metformina con acción antiinflamatoria en estudios preclínicos y la correspondiente reducción de los biomarcadores circulantes en pacientes con DM2 ${ }^{(9)}$, no existe evidencia clínica contundente de su beneficio en cuadros infecciosos virales e igualmente en pacientes con infección por coronavirus. En el contexto ambulatorio no está contraindicado su uso en el curso de un proceso infeccioso por COVID-19; sin embargo, se debe hacer seguimiento de las condiciones que puedan orientar su suspensión, como la inestabilidad y/o el compromiso de la función renal y hepática, evitando así el desarrollo de acidosis láctica.

\section{Sulfonilúreas}

Este grupo farmacológico no ofrece un diferencial como antidiabético en casos de infecciones virales agudas. No hay evidencia en cuanto al efecto deletéreo más allá de los eventos de hipoglucemia que se puedan presentar en el manejo convencional ${ }^{(2,3)}$. En este sentido, para la infección aguda por COVID-19 de seguimiento ambulatorio, se sugiere una mayor vigilancia de los episodios clínicos sugestivos de hipoglucemia.

\section{Inhibidores de la DPP-4}

Teniendo en cuenta los elementos y las cascadas fisiopatológicas descritos en el ítem correspondiente, aquel de potencial mayor trascendencia es el papel de la enzima dipeptidil peptidasa-4 (DPP-4) como receptor funcional del coronavirus, como fue documentado en el caso del hCoV-EMC, virus responsable del MERS ${ }^{(1,10)}$. Adicionalmente, como es bien conocido, dicha enzima desempeña un papel importante en el metabolismo de la glucosa, la acción de la insulina y la inflamación en los pacientes con DM2 ${ }^{(10,11)}$; sin embargo, no es contundente aún la evidencia en determinar si, en el caso de la infección por SARS-CoV-2, los inhibidores de la DPP-4 (IDPP-4) como tratamiento de la DM2 puedan tener influencia en el curso de la infección ${ }^{(1,10)}$. Desde el punto de vista pragmático, los pacientes que recibe IDPP-4 deben continuar con dosis iguales a las que venían recibiendo si el control metabólico se está cumpliendo. Los ajustes serán realizados si se presenta un compromiso agudo adicional de la función renal ${ }^{(1,3)}$. 


\section{Agonistas de los receptores de GLP-1}

Sus efectos antiinflamatorios han sido uno de los hallazgos más repetitivos y atractivos en los estudios realizados en modelos animales con este grupo farmacológico; de manera particular, se describe atenuación de la reacción inmunitaria pulmonar ${ }^{(12)} \mathrm{y}$, además, en algunos protocolos con disminución de los biomarcadores inflamatorios en pacientes con DM2 y obesidad ${ }^{(3,12,13)}$. Adicionalmente, en los estudios de seguridad de riesgo cardiovascular para los AR-GLP1 publicados en el último lustro ${ }^{(14-17)}$, no se evidenciaron proporciones diferenciales de cuadros infecciosos o de evolución infructuosa de estos. Por tanto, si bien existe la posibilidad de beneficio clínico por parte de los AR-GLP1 en procesos infecciosos, por ahora, son elucubraciones teóricas que no permiten emitir recomendaciones prácticas de preferir su elección sobre otros antidiabéticos; sin embargo, es pertinente destacar que su uso es seguro en el contexto ambulatorio de la DM2 con infección por COVID-19.

\section{Inhibidores de SGLT-2}

Este grupo farmacológico de antidiabéticos es ampliamente conocido por su perfil de beneficios cardiovasculares y renales que van más allá del control glucémico ${ }^{(18)}$. Su tolerancia en el contexto ambulatorio es adecuada; sin embargo, en el curso de procesos infecciosos agudos de evolución rápida, como es el caso de la COVID-19, se pueden evidenciar estados tempranos de deshidratación con la consecuente predisposición a complicaciones agudas (depleción de volumen y cetoacidosis euglucémica) ${ }^{(1,3)}$, motivo por el que se sugiere no suspender la medición de entrada, ya que esta conducta puede ir en detrimento del control glucémico, el cual, como se ha explicado, es fundamental; aun así, debe reevaluarse frecuentemente el estado del paciente ambulatorio infectado, con el fin de suspenderlo solo si clínicamente el paciente así lo requiere (deshidratación e inestabilidad clínica) y minimizar el riesgo de complicaciones asociadas a su uso ${ }^{(1-2,18-20)}$. Este panorama ha motivado la generación de evidencia contundente, por lo que, a la fecha, se encuentra en curso el estudio DARE-19 (Da- paglifozin in Respiratory Failure in Patients With COVID-19), un ensayo clínico de asignación aleatoria, multicéntrico, en grupos paralelos, doble ciego y controlado con placebo en pacientes hospitalizados por COVID-19, donde se evalúa el efecto de la dapagliflozina en $10 \mathrm{mg}$ frente a placebo, durante 30 días, en adición al manejo metabólico de base, en la reducción de la progresión, las complicaciones y la mortalidad por todas las causas ${ }^{(21)}$. Si bien su desarrollo es en el ambiente hospitalario, puede ofrecer luces para la toma futura de decisiones.

\section{Insulinas}

La insulina, como alternativa farmacológica en la perspectiva del manejo ambulatorio de la infección por COVID-19, pudiese estar respaldada por la atenuación de la respuesta inflamatoria de las células T, evaluada en modelos animales con influenza ${ }^{(22)}$, así como por la evidencia de su acción antiinflamatoria, evaluada con perfil de biomarcadores en estudios clínicos de pacientes agudamente enfermos ${ }^{(23)}$. Aunque en el panorama preciso de la infección por coronavirus no existe evidencia disponible, su preferencia en el ambiente hospitalario puede ser indiscutible; sin embargo, orientar al cambio de antidiabéticos por insulina ambulatoriamente no es una conducta estándar; solo se tomará si el control glucémico así lo amerita.

\section{Conflicto de interés}

Los autores certifican que no tienen afiliaciones con o participación en cualquier organización o entidad con cualquier interés financiero (como honorarios, becas educativas, participación como oradores, empleo, consultorías, propiedad de acciones u otro interés patrimonial), o interés no financiero (como relaciones personales o profesionales, afiliaciones, conocimientos o creencias) en el tema o materiales discutidos en este manuscrito.

\section{Financiación}

Los autores declaran que no recibieron recursos para la elaboración de este manuscrito. 


\section{Referencias}

1. Bornstein SR, Rubino F, Khunti K, Mingrone G, Hopkins D, Birkenfeld A, et al. Practical recommendations for the management of diabetes inpatients with COVID-19. Lancet Diabetes Endocrinol. 2020;8(6):546-50. doi: 10.1016/S2213-8587(20)30152-2.

2. Singh AK, Gupta R, Ghosh A, Misra A. Diabetes in COVID-19: Prevalence, pathophysiology, prognosis and practical considerations. Diabetes Metab Syndr. 2020:14(4):303-10. doi: 10.1016/j.dsx.2020.04.004.

3. Drucker DJ. Coronavirus Infections and Type 2 Diabetes-Shared Pathways With Therapeutic Implications. Endocr Rev. 2020;41(3):bnaa011. doi: 10.1210 /endrev/bnaa011.

4. Gupta R, Ghosh A, Singh ak, Misra a. Clinical considerations for patients with diabetes in times of COVID-19 epidemic. Diabetes Metab Syndr. 2020;14(3):211-2. doi: 10.1016/j.dsx.2020.03.002.

5. de Simone G. Position statement of the ESC Council on Hypertension on ACE-inhibitors and angiotensin receptor blockers [Internet]. [Consultado 15 de abril de 2020]. Disponible en: https://bit.ly/36IKUHl.

6. Raj VS, Mou H, Smits SL, Dekkers DHW, Müller MA, Dijkman R, et al. Dipeptidyl peptidase 4 is a functional receptor for the emerging human coronavirus-EMC. Nature. 2013;495(7440):251-4. doi: 10.1038/nature12005.

7. Andersen CJ, Murphy KE, Fernandez ML. Impact of obesity and metabolic syndrome on immunity. Adv Nutr. 2016;7(1):66-75. doi: 10.3945/ an.115.010207.

8. International Diabetes Federation Europe. How to manage diabetes during an illness? "Sick Day Rules" [Internet]. Disponible en: https://bit. ly $/ 3 \operatorname{cg} 3$ urt

9. Cameron AR, Morrison VL, Levin D, Mohan M, Forteath C, Beall C, et al Anti-Inflammatory effects of metformin irrespective of diabetes status. Circ Res. 2016;119(5):652-65. doi: 10.1161/CIRCRESAHA.116.308445.

10. Iacobellis G. COVID-19 and diabetes: Can DPP4 inhibition play a role? Diabetes Research Clin Pract. 2020;162:108125. doi: 10.1016/j.diabres.2020.108125.

11. Mulvihill EE, Drucker DJ. Pharmacology, physiology, and mechanisms of action of dipeptidyl peptidase-4 inhibitors. Endocr Rev. 2014;35(6):9921019. doi: 10.1210/er.2014-1035.

12. Zhou F, Zhang Y, Chen J, Hu X, Xu Y. Liraglutide attenuates lipopolysaccharide induced acute lung injury in mice. Eur J Pharmacol. 2016;791:735-40. doi: 10.1016/j.ejphar.2016.10.016.
13. Drucker DJ. Mechanisms of action and therapeutic application of glucagon-like peptide-1. Cell Metab. 2018;27(4):740-56. doi: 10.1016/j cmet.2018.03.001.

14. Marso SP, Daniels GH, Brown-Frandsen K, Kristensen P, Mann J, Nauck MA et al. Liraglutide and cardiovascular outcomes in type 2 diabetes. $\mathrm{N}$ Engl J Med. 2016;375(4):311-22. doi: 10.1056/NEJMoa1603827

15. Marso SP, Bain SC, Consoli A, Eliaschewitz FG, Jódar E, Leiter LA, et al. Semaglutide and cardiovascular outcomes in patients with type 2 diabetes. $\mathrm{N}$ Engl J Med. 2016;375(19):1834-44. doi: 10.1056/NEJMoa1607141.

16. Holman RR, Bethel MA, Mentz RJ, Thompson VP, Lokhnygina Y, Buse JB, et al. Effects of once weekly exenatide on cardiovascular outcomes in type 2 diabetes. N Engl J Med. 2017;377(13):1228-39. doi: 10.1056/NEJMoa1612917.

17. Gerstein HC, Colhoun HM, Dagenais GR, Diaz R, Lakshmanan M, Pais $P$, et al. Dulaglutide and cardiovascular outcomes in type 2 diabetes (REWIND): a double-blind, randomised placebo-controlled trial. Lancet 2019;394(10193):121-30, doi: 10.1016/S0140-6736(19)31149-3.

18. American Diabetes Association. 9. Pharmacologic Approaches to Glycemic Treatment: Standards of Medical Care in Diabetes-2020. Diabetes Care. 2020;43(Suppl 1):S98-S110. doi: 10.2337/dc20-S009.

19. Hamblin PS, Wong R, Ekinci EI, Fourlanos S, Shah S, Jones A, et al. SGLT2 inhibitors increase the risk of diabetic ketoacidosis developing in the community and during hospital admission. J Clin Endocrinol Metab. 2019;104(8):3077-87. doi: 10.1210/jc.2019-00139.

20. Royal United Hospitals Bath. NHS Foundation Trust. Urgent Safety Notice: Use of SGLT-2 inhibitor during the COVID-19 crisis [Internet]. Disponible en: https://www.ruh.nhs.uk/.

21. Kosiborod M. Dapagliflozin in Respiratory Failure in Patients With CO VID-19 (DARE-19) [Internet]. Disponible en: https://www.clinicaltrials gov/ct2/show/NCT04350593.

22. Tsai S, Clemente-Casares X, Zhou AC, Lei H, Ahn JJ, Chan YT, et al. Insulin receptor-mediated stimulation boosts $\mathrm{T}$ cell immunity during inflammation and infection. Cell Metab. 2018;28(6):922-934.e924. doi: 10.1016/j. cmet.2018.08.003.

23. Hansen TK, Thiel S, Wouters PJ, Christiansen JS, Van den Berghe G. Intensive insulin therapy exerts antiinflammatory effects in critically ill patients and counteracts the adverse effect of low mannose-binding lectin levels. Clin Endocrinol Metab. 2003;88(3):1082-8. doi:10.1210/jc.2002-021478. 